\title{
A Population-level Analysis of Morning Song: Exploring the Implications for Point Counts
}

\author{
Jennifer R. Foote ${ }^{1,4,5}$, Lauren P. Fitzsimmons ${ }^{2,5}$, Lynnea M. Lobert ${ }^{1}$, Laurene M. RatclifFE ${ }^{3}$, \\ and DANIEL J. MENNILL ${ }^{2}$
}

\author{
${ }^{1}$ Department of Biology, Algoma University, 1520 Queen Street East, Sault Ste. Marie, Ontario P6A 2G4 Canada \\ ${ }^{2}$ Department of Biological Science, University of Windsor, 401 Sunset Avenue Windsor, Ontario N9B 3P4 Canada \\ ${ }^{3}$ Department of Biology, Queen's University, 116 Barrie Street, Kingston, Ontario K7L 3N6 Canada \\ ${ }^{4}$ Corresponding author: jennifer.foote@algomau.ca \\ ${ }^{5}$ Authors contributed equally to the manuscript
}

Foote, Jennifer R., Lauren P. Fitzsimmons, Lynnea M. Lobert, Laurene M. Ratcliffe, and Daniel J. Mennill. 2017. A populationlevel analysis of morning song: exploring the implications for point counts. Canadian Field-Naturalist 131(1): 10-18. https://doi.org/10.22621/cfn.v131i1.1779

Point counts are widely used for conducting ecological surveys of wild birds. Vocal output of birds varies with time of day, and therefore the results of ecological surveys should also vary with time of day. We modeled how males' singing rates change over the morning. We calculated song rates in $3 \mathrm{~min}$ sampling periods (the standard sampling period used by the North American Breeding Bird Survey) and compared how detection rates vary as sampling period increases. We recorded singing activity in 15 neighbourhoods of breeding Black-capped Chickadees (Poecile atricapillus) with 16-element microphone arrays that recorded every song from every male in every neighbourhood. We calculated the proportion of males that produced one or more songs during 3,5, and 10 min count periods between nautical twilight and late morning. Our results show a strong peak in singing activity just before sunrise followed by a steady decline in singing activity over the course of the morning. We found that longer sampling periods yielded significantly higher detection rates at all times after sunrise. After sunrise, detection rates never exceeded $60 \%$, even with 10 min sampling periods. We found that unpaired males had significantly higher detection rates than paired males but we found no difference between paired males with fertile versus incubating mates. Our results provide strong evidence that, for Black-capped Chickadees, surveys during the dawn chorus provide the most comprehensive assessment of the number of birds present, and that longer sampling periods yield significantly better estimates of population sizes at all times after sunrise.

Key Words: Acoustic location system; Black-capped Chickadee; dawn song; detection rate; point count

\section{Introduction}

The heightened vocal output from birds in the early morning offers an excellent opportunity to conduct point-count surveys. The North American Breeding Bird Survey, for example, is an annual survey used to study population changes over time (Link and Sauer 1998). Point counts are used by many agencies around the globe to estimate the abundance and diversity of birds (e.g., BirdLife International/European Bird Census Council 2000; Urfi et al. 2005). Breeding Bird Surveys take place in the morning, between $30 \mathrm{~min}$ before sunrise and approximately $4 \mathrm{~h}$ after sunrise. At each stop all birds seen or heard within $400 \mathrm{~m}$ are counted during a $3 \mathrm{~min}$ interval (Ziolkowski Jr. et al. 2010). Survey data are widely used in analyses of bird population numbers, providing targets for conservation initiatives (Link and Sauer 1998). Surveys usually rely on aural detection (Brewster and Simons 2009), yet birds' vocal output varies throughout the day, and therefore detectability varies with the timing of point counts (Farnsworth et al. 2002; Alldredge et al. 2007). Population estimates using Breeding Bird Survey data often apply time-of-day adjustments to account for differences in detection rates over the course of a survey day
(Rosenberg and Blancher 2005). Corrections for timeof-day take into account peak numbers on a route and adjust average numbers to peak numbers simulating peak numbers continuing throughout the morning. The number of detected birds is then doubled assuming a single detected bird represents a pair (Rosenberg and Blancher 2005). Unfortunately, there are relatively few studies of diel singing rates to test the time-of-day adjustment values calculated from survey data, which may lead to inaccurate estimates of population size (Thogmartin 2010).

Point count survey length is positively correlated with the number of species detected and the number of birds detected (Scott and Ramsay 1981; Fuller and Langslow 1984). Recently, Matsuoka et al. (2014) assessed recommendations for survey length and survey detection distances and found problems with current techniques. In most surveys, one or both of the distance standards (e.g., $50 \mathrm{~m}$ radius and unlimited radius) or time standards (e.g., $0-3 \mathrm{~min}$ followed by $3-5$ min intervals) were not applied. For studies that collected data during more than one time frame (e.g., a 3 min survey followed immediately by an additional 2 min survey), an increased survey length resulted in $25-65 \%$ higher detec- 
tion rates (Matsuoka et al. 2014). Matsuoka et al. (2014) call for a return to previously recommended standards to improve the quality of survey data to allow for meaningful comparisons across studies and metaanalyses. Specifically, Matsuoka et al. (2014) argued that surveyors should record birds at two spatial scales (within $50 \mathrm{~m}$ of the observer and beyond $50 \mathrm{~m}$ from the observer) and two or three time scales (within a $0-3$ min sampling window followed by additional 2 and 5 min sampling windows). Thompson (2002) highlighted the importance of testing detectability assumptions of count data using marked populations where estimates can be compared to true-population sizes, although this recommendation has rarely been implemented.

In this study, we performed analyses of singing behaviour in a breeding population of individuallymarked Black-capped Chickadees (Poecile atricapillus) using a 16-microphone array. We monitored our study population intensively over a six-month period from mid-winter until after the breeding season, and therefore we knew the exact number of animals in the recorded areas and the identities of each of the singers. Given that we knew the total number of males in each area where we collected recordings, we were able to quantify what proportion of the population was singing at regular sampling intervals across the morning. Our first objective was to assess how the number of singing males varied temporally over the course of the morning, and to calculate a time-of-day adjustment for Blackcapped Chickadees using data from continuous passive acoustic sampling of their vocal behaviour. Our second objective was to examine how aural detection rates changed when the survey length increased from $3 \mathrm{~min}$ to $5 \mathrm{~min}$ and $10 \mathrm{~min}$, and how those detection rate differences changed over the course of the morning. Our third and final objective was to evaluate how detection rates varied with the pairing status (bachelor males versus paired males) and the breeding status (males with fertile females versus males with incubating females) of the singers.

\section{Methods}

We studied a banded population of Black-capped Chickadees at the Queen's University Biological Station $\left(44.567^{\circ} \mathrm{N}, 76.317^{\circ} \mathrm{W}\right)$, north of Kingston, Ontario, Canada from January to July, 2005-2007. Adult birds were captured in winter using treadle-traps baited with sunflower seeds. We banded adult Black-capped Chickadees with aluminum Canadian Wildlife Service bands as well as unique combinations of coloured leg bands (newly banded birds: $n=149$ in 2005, $n=236$ in 2006, $n=61$ in 2007). Beginning in late April, we recorded dawn and morning songs of male Black-capped Chickadees using a custom built 16-microphone Acoustic Location System. For a detailed description of the Acoustic Location System, see Fitzsimmons et al. (2008).
Each 16-channel microphone array recorded a neighbourhood of males, encompassing an area of 160 000 $\mathrm{m}^{2}$ (e.g., $400 \mathrm{~m} \times 400 \mathrm{~m}$ ). A "neighbourhood" consisted of a cluster of 5 to 10 breeding territories (6.66 \pm 1.34 ; mean \pm SE) occupied by birds that were familiar with each other from their previous winter flock or nearby flocks. We focused our analysis on the males within each neighbourhood that had territories located centrally within the array of recording areas (32 males in 5 arrays in 2005, 34 males in 5 arrays each in 2006 and 2007). In the current analysis, we did not include males on the edge of our array recording areas (i.e., males whose territories were not entirely encompassed by our microphones) because we could not be certain that we recorded all of their songs. From 30 April to 14 May, 2005-2007, we recorded 15 chickadee neighbourhoods (five in each year) for 2-3 mornings per neighbourhood. Recordings began just before or at nautical twilight and continued throughout the morning until approximately 11:00 EST. Daily recordings averaged $365.9 \pm 2.4 \mathrm{~min}$ in length.

Our analyses relied on acoustic monitoring of songs that are produced by male Black-capped Chickadees (Foote et al. 2010), and are the most common vocalization of this species during the breeding season. In contrast to the solely acoustic focus of our surveys, Breeding Bird Surveys also include visual detections in addition to acoustic detections. Yet given that bird detections are primarily aural during Breeding Bird Surveys (Brewster and Simmons 2009), and numbers are then doubled to determine the number of breeding individuals, we believe our acoustic data are comparable (Rosenberg and Blancher 2005).

We used Syrinx-PC sound analysis software (J. Burt, Seattle, Washington, USA) to view and annotate 16channel spectrograms of array recordings. We selected one morning of recording from each neighbourhood and we annotated all chickadee songs using the frequency and time cursors in Syrinx-PC. Although point counts often focus on both songs and calls, we chose to focus on songs in this analysis, given that songs are the most conspicuous, far-reaching vocalization produced by Black-capped Chickadees during the breeding season. We used a combination of field notes and location information to identify singing males (as in Fitzsimmons et al. 2008; Foote et al. 2008). During the recording period, 3 to 5 observers took detailed notes of the time and location of males producing songs, identifying as many colour-banded birds as possible. During annotation of sound files, the identity and location of each singing male was approximated using time of arrival distances at the different recording channels, as well as song amplitude differences between the different channels. If male identity was ambiguous when comparing the approximate position to our field notes, we used ARRAYGUI in MATLAB (see Mennill et al. 2006) to triangulate the position of the singing bird to identify it. In addition, we regularly compared the fine- 
structural features of the array-recorded songs to reference recordings we collected from males in the field; subtle differences in the spectral properties of male songs facilitate identification from spectrograms. The output from the annotation process in Syrinx-PC was a spreadsheet containing a time-stamped record of all songs produced by all males during recording. From these spreadsheets, we calculated the number of unique males singing in each $3 \mathrm{~min}, 5 \mathrm{~min}$, and $10 \mathrm{~min}$ period, using rolling (overlapping) time bins. For each neighbourhood, we determined how many males held territories within the recorded area, and then calculated the proportion of singing males during sampling intervals relative to the total number of males in the neighbourhood. For males that we knew to be bachelors during the recordings ( $n=5$ males across the 15 recordings), we calculated the proportion of males singing in each 3 min interval, and we did the same for five randomly-chosen paired males for comparison. For 12 males from 2005 with confirmed nesting stage on the day of recording, we calculated the proportion of males singing for six males with a fertile mate on the day of recording (we determined fertile period as $2 \mathrm{~d}$ prior to egg laying until the penultimate egg was laid; Smith 1991) and the proportion of males singing for six males with incubating mates. We standardized the timing of songs by calculating them relative to morning nautical twilight. The absolute time of day would not be appropriate given that the timing of sunrise changes daily. We calculated the time of nautical twilight based on data for the nearest city (Kingston, Ontario, Canada, approximately $50 \mathrm{~km}$ due south) from the National Research Council of Canada (2014) Sunrise/Sunset Calculator.

We used logistic regression to analyze variation in the mean proportion of males singing over the course of the morning. The fixed factor was time (min past nautical twilight) and we ran separate regressions for the three sampling period lengths $(3 \mathrm{~min}, 5 \mathrm{~min}$, and $10 \mathrm{~min})$. We calculated a time-of-day adjustment factor according to the methods described in Rosenberg and Blancher (2005); the adjustment is the ratio of peak detections to mean detections across the morning. To compare detection rates as a function of the length of the sampling window, we compared the proportion of males recorded in $3 \mathrm{~min}$ periods to the proportions sampled in $5 \mathrm{~min}$ and $10 \mathrm{~min}$ periods using Wilcoxon Signed-Rank tests. To compare detection rates of paired and unpaired males and males with fertile and incubating mates, we calculated the mean proportion of males detected in 3 min count periods during each 30 min interval between nautical twilight and the time when the first of these males stopped singing (317 min after twilight). We used a linear model on log-transformed detection probabilities to test for an effect of time, pairing status or fertile stage, and an interaction between the two factors. For this analysis, we excluded the first data point, $30 \mathrm{~min}$ after twilight when most birds had not yet begun to sing. We analysed data using JMP 11 (SAS Institute, Cary, North Carolina, USA). Values are reported as means $\pm \mathrm{SE}$, and all tests were two-tailed.

\section{Results}

Our analyses of morning-long recordings of 15 neighbourhoods of Black-capped Chickadees reveal that the detection probability for males reached a peak prior to sunrise, and then declined steadily over the course of the morning (logistic regression: $\chi_{1}^{2}=113.6$, $P<0.001$; Figure 1). The proportion of males singing was highest during the dawn chorus, before sunrise, when approximately $90 \%$ of males in the population produced song. The time-of-day correction factor was 3.6, calculated as the ratio of peak detections to mean detections, calculated from continuous sampling across the 15 Black-capped Chickadee neighbourhoods.

Lengthening the sampling window from $3 \mathrm{~min}$ to $5 \mathrm{~min}$ or $10 \mathrm{~min}$ led to a significant increase in the total proportion of males detected (3 versus 5 min: Wilcoxon test: $W_{80}=1463.5, P<0.001 ; 3$ versus $10 \mathrm{~min}$ : Wilcoxon test: $W_{37}=370.5, P<0.001$; Figure 2). Increasing from $3 \mathrm{~min}$ to $5 \mathrm{~min}$ periods resulted in 24.4 $\pm 2.5 \%$ more males detected, and increasing from 3 min to $10 \mathrm{~min}$ periods resulted in $69.7 \pm 6.4 \%$ more males detected.

The proportion of males that were detected during longer sampling periods increased over the course of the morning (logistic regression: 3 versus 5 min: $\chi_{1}^{2}$ $=10.6, P=0.0011 ; 3$ versus $10 \mathrm{~min}: \chi_{1}^{2}=11.51, P=$ 0.0007; Figure 3; Table 1). However, after sunrise the proportion of males detected never exceeded $60 \%$ of all of the males in the neighbourhood, even with longer sampling periods of 5 or $10 \mathrm{~min}$.

The proportion of unpaired males detected in $3 \mathrm{~min}$ sampling periods was higher after sunrise than the proportion of paired males detected (linear model: $r^{2}=$ $0.65, F_{2,17}=15.63, P=0.0001$; Figure 4$)$. Both pairing status $\left(F_{1,18}=8.64, P=0.0091\right)$ and time-of-day $\left(F_{1,18}\right.$ $=22.62, P=0.0002)$ were significantly related to detection probability; however, the interaction between time and pairing status was not significant and was not retained in the model. In 3 min sampling periods, we detected on average $40.0 \pm 0.07 \%$ of unpaired males and $27.0 \pm 0.07 \%$ of paired males. The proportion of males detected with fertile or incubating mates did not show a clear pattern (linear model: $r^{2}=0.50, F_{2,17}=$ 8.52, $P=0.0027$; Figure 5). Time-of-day $\left(F_{1,18}^{2,17}=\right.$ $3.94, P=0.0010$ ) was significantly related to detection probability (as above); however, pairing status did not relate to probability of detection $\left(F_{1,18}=1.22, P\right.$ $=0.24$ ) and the interaction between time of day and pairing status was not significant and was not retained in the model. In 3 min sampling periods, we detected on average $31.1 \pm 0.08 \%$ of males with fertile mates and $36.0 \pm 0.08 \%$ of males with incubating mates. 


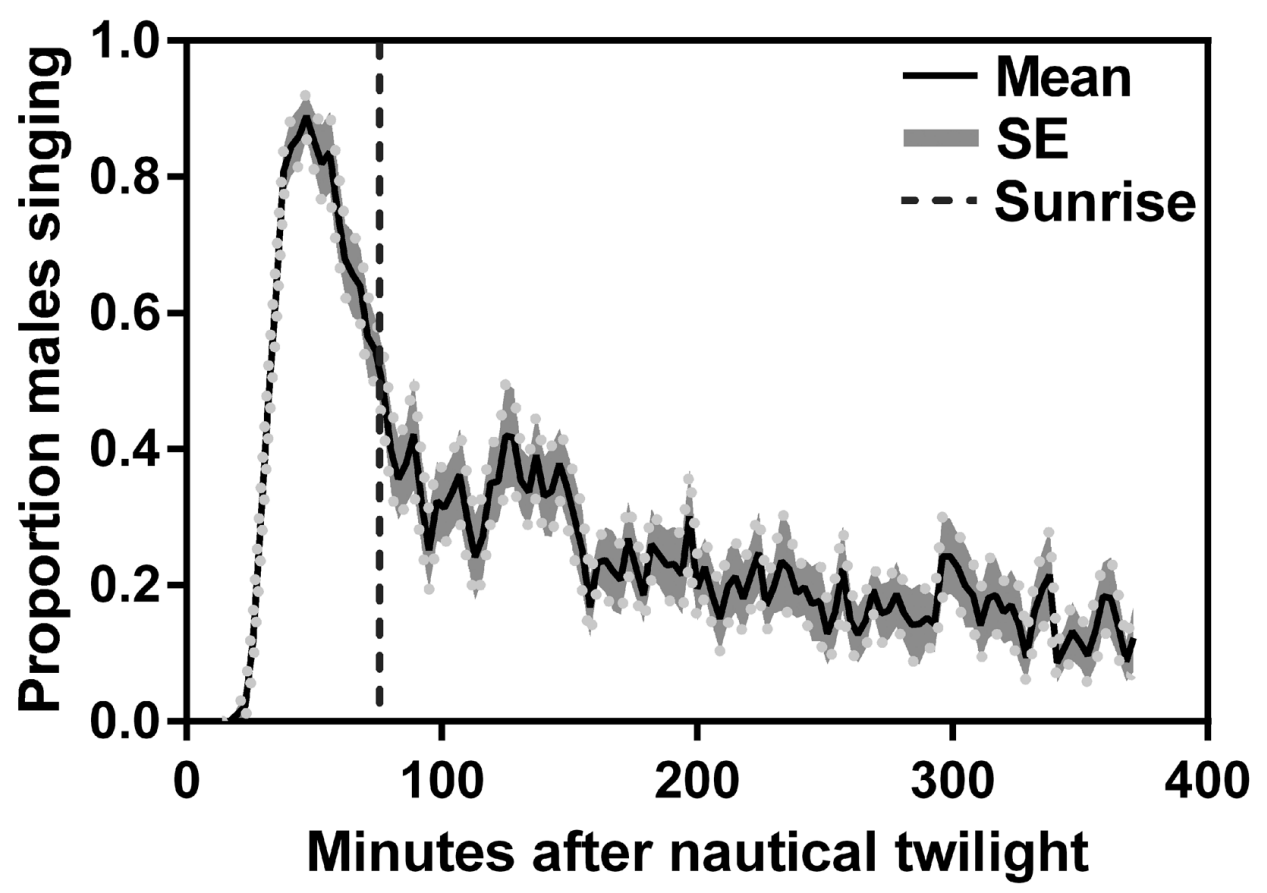

FIGURE 1. The mean ( $\pm \mathrm{SE}$ ) proportion of male Black-capped Chickadees (Poecile atricapillus) singing in 3 min sampling periods from nautical twilight through late morning.

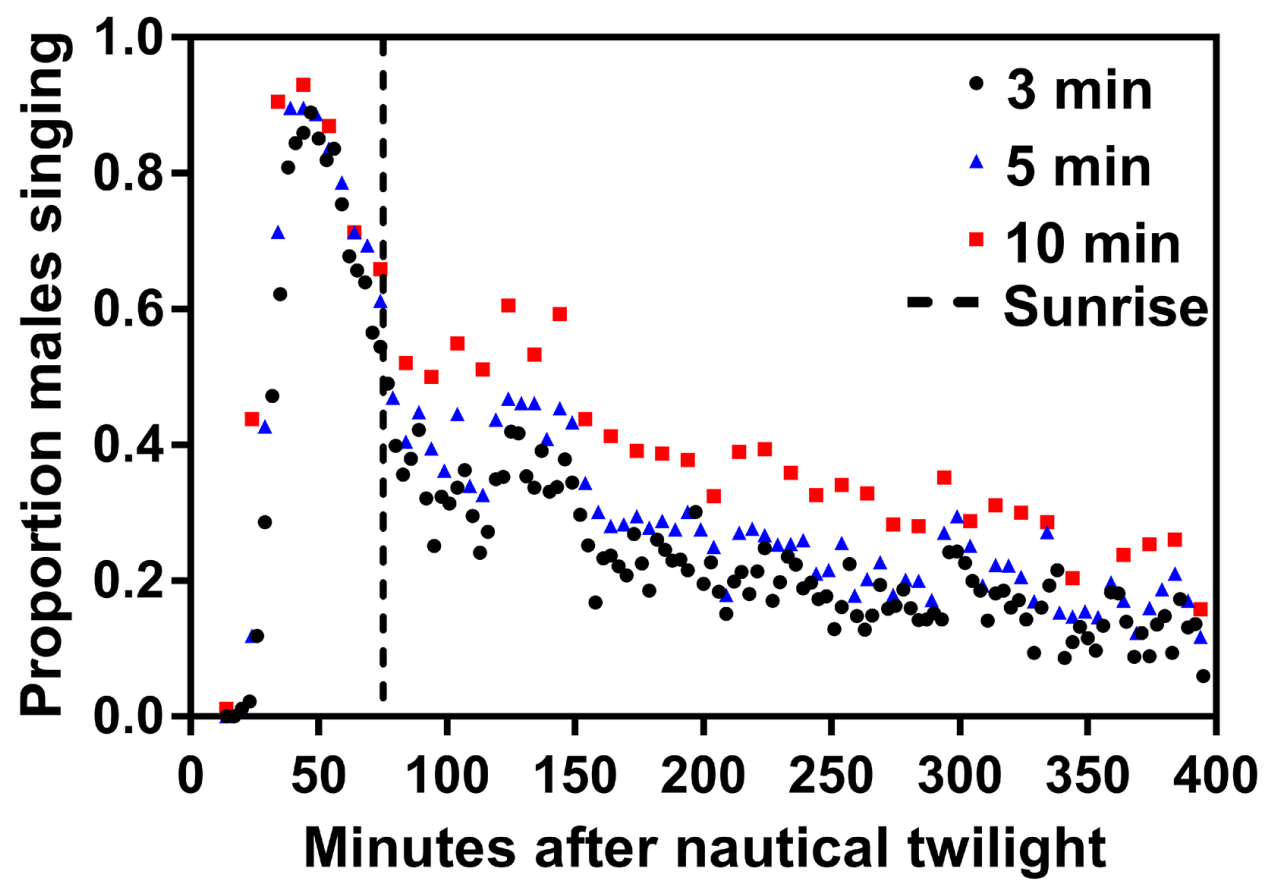

FIGURE 2. A comparison of detection rates of Black-capped Chickadees (Poecile atricapillus) with 3 min sampling periods (circle), 5 min periods (triangle), and 10 min periods (square) from nautical twilight through late morning. 


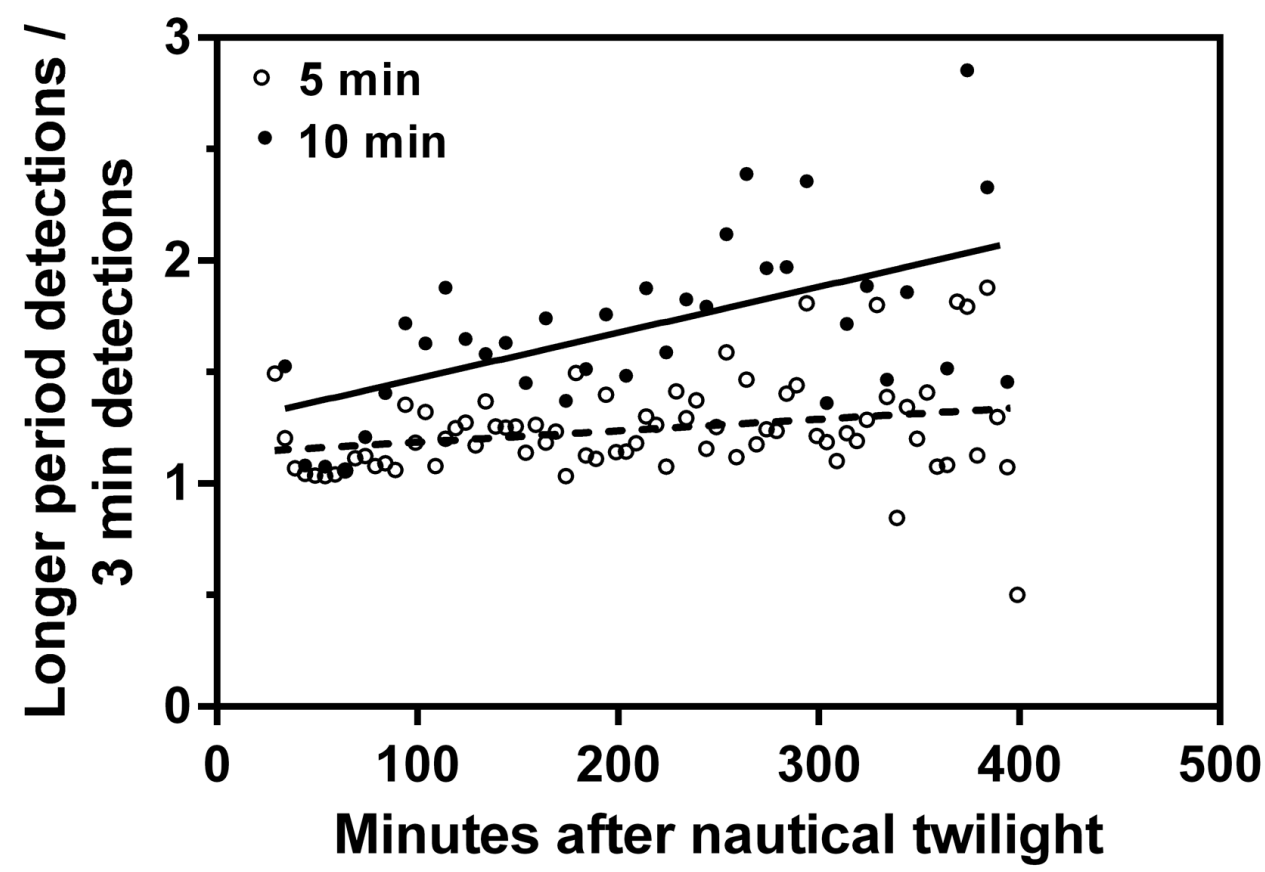

FIGURE 3. A comparison of detection rates of Black-capped Chickadees (Poecile atricapillus) between 3 min periods and 5 and $10 \mathrm{~min}$ periods. Points are the proportion of males detected during $5 \mathrm{~min}$ (open circles) and $10 \mathrm{~min}$ (filled circles) periods divided by the proportion of males detected during 3 min periods. Improved detection rates with longer sampling periods increase over the course of the morning.

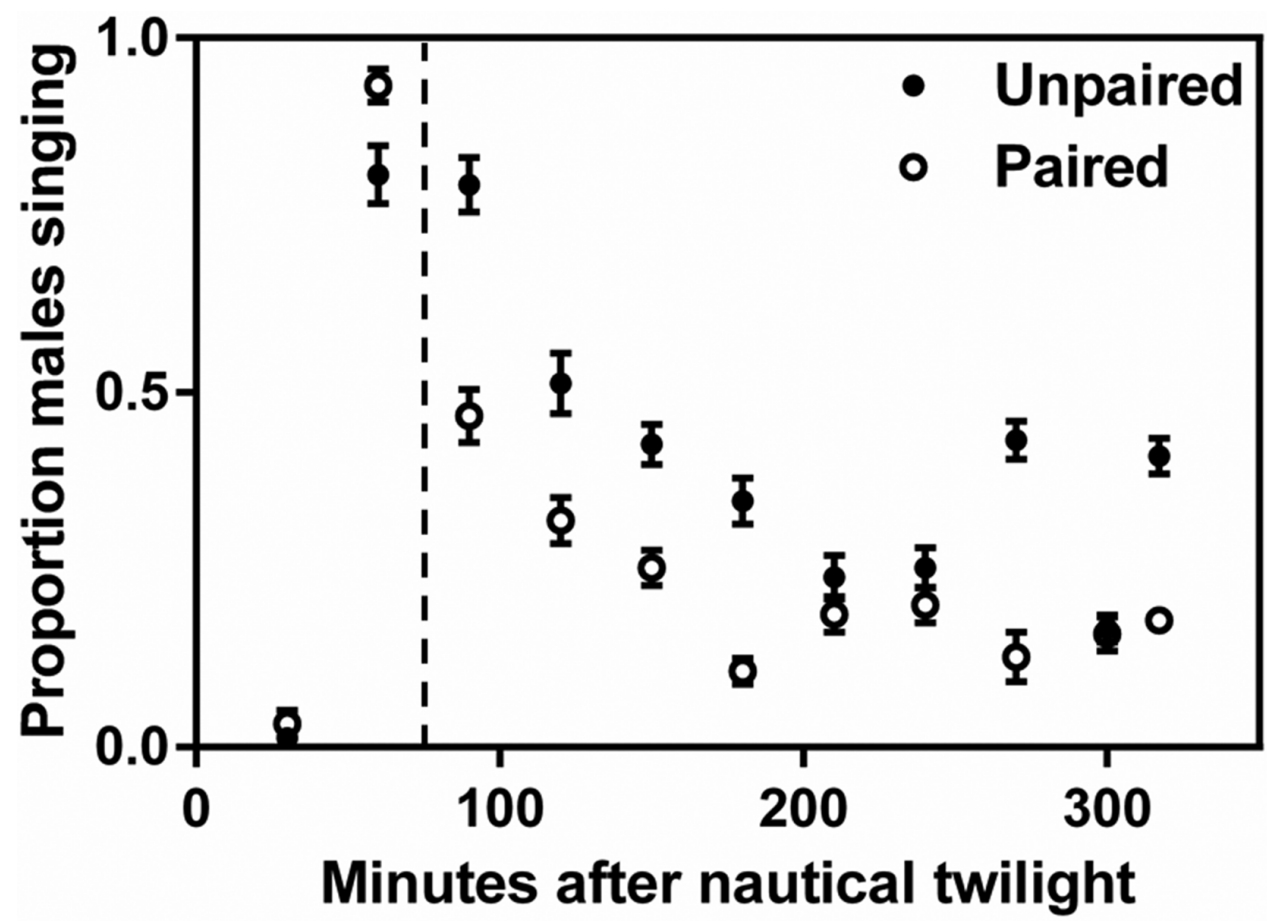

Figure 4. A comparison of detection rates of Black-capped Chickadees (Poecile atricapillus) during 3 min periods for unpaired males and paired males. Points represent the mean $( \pm \mathrm{SE})$ proportion of males detected during $30 \mathrm{~min}$ bins from nautical twilight for $317 \mathrm{~min}$ (final bin is $17 \mathrm{~min}$ long) for five unpaired males (closed circles) and five paired males (open circles). The dotted vertical line shows the time of sunrise. 
TABLE 1. Total number of Black-capped Chickadee (Poecile atricapillus) detections for first 3 min $\left(\mathrm{X}^{1}\right)$, subsequent $2 \mathrm{~min}\left(\mathrm{X}^{2}\right)$, and subsequent $5 \mathrm{~min}\left(\mathrm{X}^{3}\right)$ of $10 \mathrm{~min}$ count periods, total detections $(\mathrm{X})$ and total possible detections $(\mathrm{N})$ in each of three morning periods.

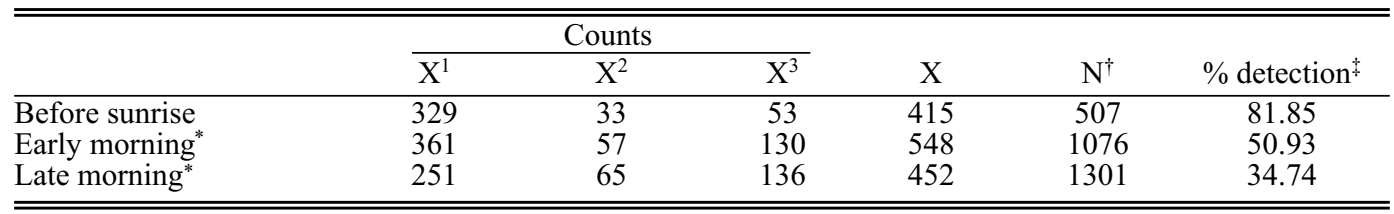

${ }^{*}$ Following methods of Farnsworth et al. (2002), early morning is sunrise to 7:45am EST and late morning is 7:46 to $10: 00$ am EST.

Total possible detections is the sum of the number of males in the population in each 10 min interval.

$+\mathrm{X} / \mathrm{N} \times 100$.

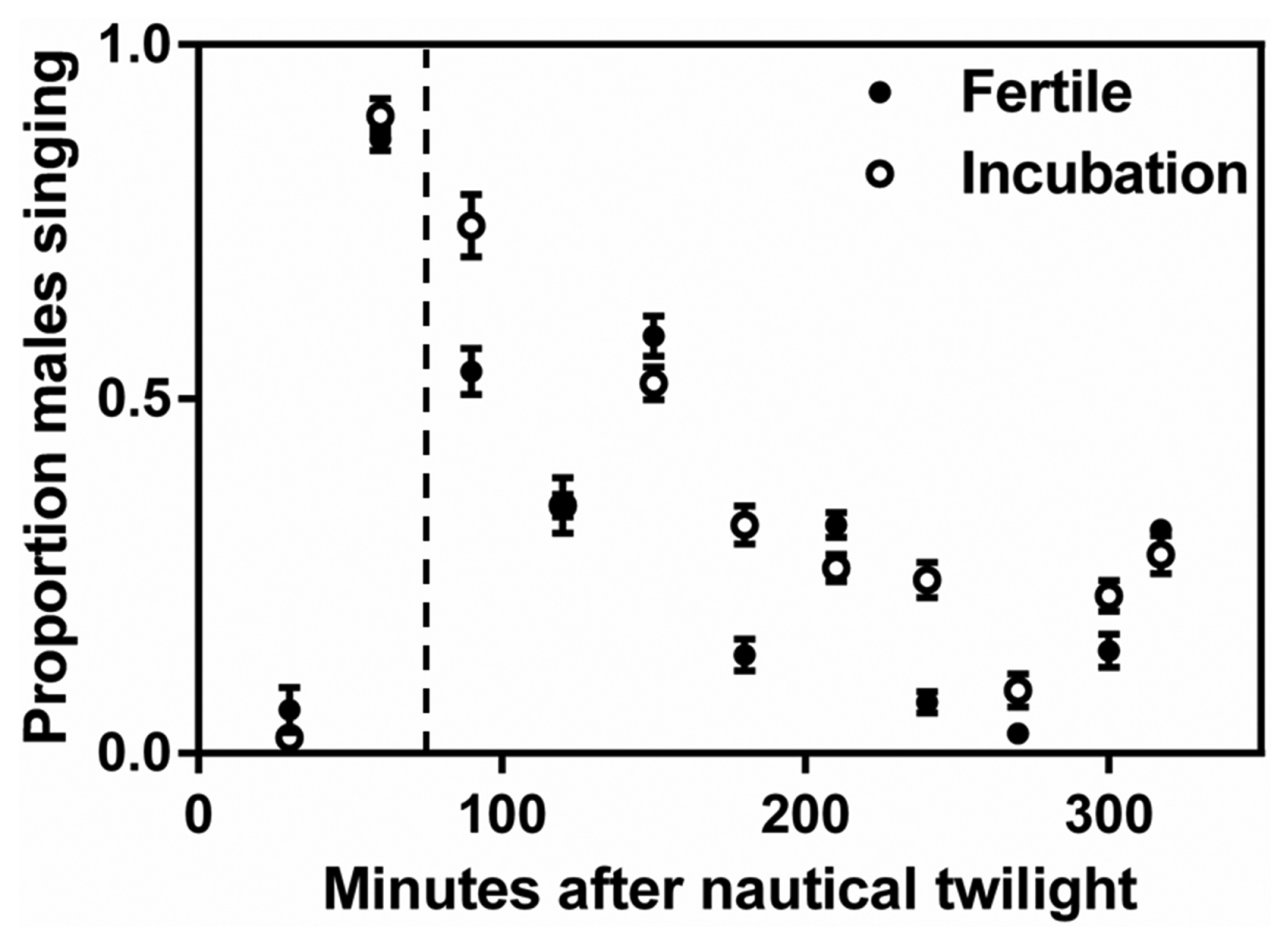

FIGURE 5. A comparison of detection rates during 3 min periods for male Black-capped Chickadees (Poecile atricapillus) with fertile mates and males with incubating mates. Points represent the mean $( \pm \mathrm{SE})$ proportion of males detected during $30 \mathrm{~min}$ bins from nautical twilight for $317 \mathrm{~min}$ (final bin is $17 \mathrm{~min}$ long) for six males with fertile females (closed circles) and six males with incubating females (open circles). The dotted vertical line shows the time of sunrise.

\section{Discussion}

Microphone array recordings of 15 neighbourhoods of Black-capped Chickadees revealed a peak in singing activity before dawn, followed by a sharp decline in singing activity after dawn, followed by a more gradual decline over the course of the morning. Extending survey periods from the standard $3 \mathrm{~min}$ period to $5 \mathrm{~min}$ and 10 min periods resulted in $24 \%$ and $70 \%$ more males detected, respectively. The proportion of males that were detected during longer sampling periods increased over the course of the morning, but even with longer surveys, detection rates after sunrise never exceeded $60 \%$ of the number of birds in the sampling area, and fell as low as $20 \%$ later in the morning. Unpaired males were detected $13 \%$ more often than were paired males in 3 min sampling periods, whereas males with fertile mates versus incubating mates were detected with similar frequency. Taken together, our results demonstrate that the most effective surveys can be conducted early in the morning (during the dawn chorus), and that sampling periods of 5 or $10 \mathrm{~min}$ provide a clear advantage over the standard 3 min period. Our results confirm the 
importance of accounting for time of day when analysing and comparing survey data, and highlight that acoustic surveys sample an increasingly small subset of the population as the morning progresses.

\section{Dawn versus daytime detectability}

Black-capped Chickadees frequently engage in countersinging interactions with territorial neighbours, both at dawn (Foote et al. 2008, 2010) and later in the morning (Fitzsimmons et al. 2008). After sunrise, countersinging interactions may occur sporadically across the early morning period and our data show that song rates are much lower post-dawn. A decline in singing activity after the dawn chorus is seen in a number of other species with a distinct dawn chorus, with song rates ranging from $1-10 \%$ of the dawn maximum in an Australian community, for example (Keast 1985). Therefore, the pattern we observed with much higher output during the dawn chorus period is not specific to Blackcapped Chickadees; this pattern is observed across diverse species (reviewed in Staicer et al. 1996). After dawn, our analyses reveal a steady decline in song rates and detection probabilities of Black-capped Chickadees. Given that we knew the population size in our recordings, we were able to calculate the exact detection probabilities; detection rates declined from $82 \%$ before sunrise, to $51 \%$ in early morning, to $35 \%$ in late-morning (Table 1). Similarly, Farnsworth et al. (2002; Table 1) found that thrushes and some warblers had lower detection probabilities in late morning compared to early morning, although vireos and other warblers did not.

\section{Time-of-day correction factors}

Time-of-day corrections calculated from focal studies have the potential to improve population estimates based on survey data, if we assume that all species exhibit a similar decline in detection probability as the morning progresses. We calculated a time-of-day adjustment value of 3.6 for Black-capped Chickadees in our study population. While we could not find a published value for Black-capped Chickadees, the median time-of-day correction value for diurnal landbird species calculated from North American Breeding Bird Survey data is 1.32 , with a range from 1.04 to 22.3 (Rosenberg and Blancher 2005). Our correction value is higher than the published median, which indicates that our study yielded a higher ratio of peak detection rate to mean detection rate. A higher time-of-day correction factor for Black-capped Chickadees suggests that their abundance could be underestimated from Breeding Bird Survey data using a general landbird correction factor.

Our time-of-day correction could be higher than the median landbird value due to our continuous sampling with acoustic recorders providing a wider range of detection rates and/or higher peak detection rates before sunrise. However, the timing of our acoustic surveys coincides nicely with the timing of Breeding Bird Sur- vey routes that are typically completed in $4 \mathrm{~h}$ beginning $30 \mathrm{~min}$ before sunrise by stopping at sites one after the other (Rosenberg and Blancher 2005; Ziolkowski Jr. et al. 2010). We recorded birds over three weeks during the peak of their breeding activity (early-mid May), whereas Breeding Bird Surveys sample birds over a longer six week period later in the season (end of May to early July). Black-capped Chickadees are early breeders (first egg date last week of April-first week of May at our study site; Foote et al. 2010), and thus detection probability based on song would likely be equal or lower in early to late season comparisons. However, this is more likely to lead to a higher, or at least equal, peak to average detection ratio later in the season because daytime song rates are likely higher during the early part of the breeding season compared to later in the season. Farnsworth et al. (2002) found that detection probability did not vary between early and late season for three of four focal species, with the fourth species displaying lower detection probability in early spring compared to late spring. Thus, the period of sampling in our study is likely representative of detectability across the spring. In two other studies of temperate songbirds, one species (Florida Grasshopper Sparrows, Ammodramus savannarum floridanus) showed variation in detection rates from day to day but did not vary synchronously across sites, whereas another species (Canada Warblers, Cardellina canadensis) did not show a strong influence of season (Hochachka et al. 2009; Demko 2012). Future studies should test seasonal shifts in detection probability as well as timeof-day shifts to determine if seasonal correction factors would also have utility. The discrepancy between the median diurnal landbird time-of-day correction factor and our results suggests that establishing species-specific correction factors is a worthwhile goal for future investigations. Incorporating time-of-day and date-specific detection rates for these species could be instrumental in refining population estimates. Automated recording systems could be used to create time-of-day correction factors for point counts given that point count detections are primarily aural. While the process of estimating species-specific correction factors may be labour intensive, it may be particularly useful for species-at-risk or species with relatively low density.

\section{Survey length}

We found that lengthening the sampling period provided better detection probabilities, matching closely the mean values calculated from surveys of 54 species reported in Matsuoka et al. (2014). However, the higher detection rates were not proportional to the longer sampling periods. This begs the question as to whether additional time spent sampling is worthwhile? North American Breeding Bird Survey routes, for example, use 3 min periods and make 50 stops. If the sampling periods were increased to 5 or $10 \mathrm{~min}$ each, it would have the effect of lowering the number of stops by $50 \%$ or more, which does not seem like a wise trade-off in 
the interest of survey sampling at the continental scale as with the Breeding Bird Survey. For other surveys, though, Matsuoka et al. (2014) recently called for a unified return to previously recommended standards, including sampling in $3 \mathrm{~min}, 5 \mathrm{~min}$, and $10 \mathrm{~min}$ periods during surveys. For traditional point-count surveys, these longer sampling periods require more time spent at each sampling location. They thus require more surveyors or more time to sample the same number of locations as shorter surveys but may result in more species detected as well as refined abundance estimates. Passive acoustic monitoring involves the continuous recording of an area that allows us to choose multiple subintervals of count period within the recordings, as suggested, without extra effort on the ground.

\section{Social factors and detectability}

We found that unpaired males were detected at significantly higher levels than paired males. Males sing to attract mates and also to defend territories against territorial rivals (Catchpole and Slater 2003) and, consequently, song rates of unpaired males are higher after sunrise compared to males that are already paired (reviewed by Møller 1991). There are few studies that directly test this effect on the detectability of birds during surveys. Similar to our results for Black-capped Chickadees, two other studies of temperate songbirds (Common Nightingales, Luscinia megarhynchos; Canada Warblers) show that unpaired males have a higher detection probability compared to paired males (Amrhein et al. 2007; Demko 2012). A third study compared population density estimates derived from aural detections for paired and unpaired males; in two temperate songbirds (Ovenbirds, Seiurus aurocapilla; Kentucky Warblers, Geothlypis formosa) $100 \%$ of unpaired males were detected but less than $65 \%$ of paired males were detected (Gibbs and Wenny 1993). Our results show that after sunrise, unpaired male Blackcapped Chickadees are more likely to be detected than paired males (Figure 4). Although Breeding Bird Surveys are designed to estimate the number of breeding pairs, these collected findings demonstrate that acoustic surveys may overestimate the number of reproductive individuals when unpaired males are more likely to be detected. Thus, data on sex-ratio biases or the prevalence of unpaired territorial males may be an important factor to include in survey analyses. Unpaired territorial male Black-capped Chickadees are uncommon in our study population, however (five of 100 males in this study), and therefore we anticipate that pairing status would have only a small influence on abundance estimates. Species with higher sex-ratio biases with many singing unpaired males could have exaggerated abundance estimates due to the high probability of detecting unpaired individuals.

We found no obvious pattern of variation in detectability with breeding stage (males with fertile breeding partners versus incubating breeding partners). This suggests that early breeding stage has a negligible influ- ence on detection rates in Black-capped Chickadees, despite lower overall song rates at dawn in males with fertile partners (Foote et al. 2008). However we did not consider later breeding stages. Within a species, levels of extra-pair paternity (Suter et al. 2009), paternal care (e.g., Stoehr and Hill 2000), mate guarding (Johnsen et al. 2003), breeding synchrony (van Dongen 2008), territory density (e.g., Sexton et al. 2007), and pairing status (e.g., Hennin et al. 2009) can result in substantial variation in song output. It would be interesting to better understand whether consistent singing patterns exist across species with similar life-history traits such that correction values could vary with these factors.

\section{Acknowledgements}

We thank J. Baldock, T. Barran, R. Bull, S. Doucet, D. Gabriel, H. Hennin, R. Jamieson, S. Lippold, A. McKellar, A. Osmun, D. Potvin, C. Toth, and K. Winger for assistance with field work. We thank the Curtis, Lundell, Warren, Weatherhead-Metz, and Zink families for access to property, and the staff of Queen's University Biological Station for logistical support. We thank J. Burt for software design. We thank Charles Francis, two anonymous reviewers, and the Associate Editor for comments that improved the manuscript. This research was funded by the Natural Science and Engineering Research Council of Canada, the Canada Foundation for Innovation, the Ontario Government, the Society of Canadian Ornithologists, the American Ornithologists Union, the American Museum of Natural History, the University of Windsor, Queen's University, and the Algoma University Research fund.

\section{Literature Cited}

Alldredge, M. W., T. R. Simons, and K. H. Pollock. 2007. Factors affecting aural detections of songbirds. Ecological Applications 17: 948-955. https://doi.org/10.1890/06-0685

Amrhein, V., H. P. Kunc, R. Schmidt, and M. Naguib. 2007. Temporal patterns of territory settlement and detectability in mated and unmated Nightingales Luscinia megarhynchos. Ibis 149: 237-244. https://doi.org/10.1111 /j.1474-919X.2006.00621.x

BirdLife International/European Bird Census Council. 2000. European bird populations: estimates and trends. BirdLife Conservation Series No. 10. BirdLife International, Cambridge, UK.

Brewster, J. P., and T. R. Simons. 2009. Testing the importance of auditory detections in avian point counts. Journal of Field Ornithology 80: 178-182. https://doi.org/10.1111 /j.1557-9263.2009.00220.x

Catchpole, C. K., and P. J. Slater. 2003. Bird Song: Biological Themes and Variations. Cambridge University Press, Cambridge, UK. https://doi.org/10.1017/CBO9780511754791

Demko, A. 2012. Temporal and individual song variation in the Canada Warbler (Cardellina canadensis). M.Sc. thesis, Dalhousie University, Halifax, Nova Scotia, Canada.

Farnsworth, G. L., K. H. Pollock, J. D. Nichols, T. R. Simons, J. E. Hines, and J. R. Sauer. 2002. A removal model for estimating detection probabilities from pointcount surveys. Auk 119: 414-425. https://doi.org/10.2307 /4089888 
Fitzsimmons, L. P., J. R. Foote, L. M. Ratcliffe, and D. J. Mennill. 2008. Frequency matching, overlapping and movement behaviour in diurnal countersinging interactions of black-capped chickadees. Animal Behaviour 75: 19131920. https://doi.org/10.1016/j.anbehav.2007.11.006

Foote, J. R., L. P. Fitzsimmons, D. J. Mennill, and L. M. Ratcliffe. 2008. Tied to the nest: male black-capped chickadees decrease dawn chorus movement behaviour when their mate is fertile. Animal Behaviour 76: 1227-1233. https://doi.org/10.1016/j.anbehav.2007.11.006

Foote, J. R., L. P. Fitzsimmons, D. J. Mennill, and L. M. Ratcliffe. 2010. Black-capped chickadee dawn choruses are interactive communication networks. Behaviour 147: 1219-1248. https://doi.org/10.1163/000579510X513761

Fuller, R. J., and D. R. Langslow. 1984. Estimating numbers of birds by point counts: how long should counts last? Bird Study 31: 195-202. https://doi.org/10.1080/00063658409 476841

Gibbs, J. P., and D. G. Wenny. 1993. Song output as a population estimator: effect of male pairing status. Journal of Field Ornithology 64: 316-322.

Hennin, H.L., N. K. Barker, D. W. Bradley, and D. J. Mennill. 2009. Bachelor and paired male rufous-and-white wrens use different singing strategies. Behavioral Ecology and Sociobiology 64: 151-159. https://doi.org/10.1007/s00265009-0832-4

Hochachka, W. M., M. Winter, and R. A. Charif. 2009. Sources of variation in singing probability of Florida grasshopper sparrows, and implications for design and analysis of auditory surveys. Condor 111: 349-360. https://doi.org/10.15 25/cond.2009.080086

Johnsen, A., J. T. Lifjeld, and C. Krokene. 2003. Agerelated variation in mate-guarding intensity in the bluethroat (Luscinia s. svecica). Ethology 109: 147-158. https://doi.org/10 .1046/j.1439-0310.2003.00861.x

Keast, A. 1985. Temporal vocalization patterns in members of a eucalypt forest bird community: the effects of weather on song production. Emu 94: 172-180. https://doi.org/10.1071 /MU9940172

Link, W. A., and J. R. Sauer. 1998. Estimating population change from count data: application to the North American Breeding Bird Survey. Ecological Applications 8: 258268. https://doi.org/10.1890/1051-0761(1998)008\%5B 0258:EPCFCD\%5D2.0.CO;2

Matsuoka, S. M., C. L. Mahon, C. M. Handel, P. Sólymos, E. M. Bayne, P. C. Fontaine, and C. J. Ralph. 2014. Reviving common standards in point-count surveys for broad inference across studies. Condor 116: 599-608. https://doi .org/10.1650/CONDOR-14-108.1

Mennill, D. J., J. M. Burt, K. M. Fristrup, and S. L. Vehrencamp. 2006. Accuracy of an acoustic location system for monitoring the position of duetting tropical songbirds. Journal of the Acoustical Society of America 119: 2832 2839. https://doi.org/10.1121/1.2184988
Møller, A. P. 1991. Why mated songbirds sing so much: mate guarding and male announcement of mate fertility status. American Naturalist 138: 994-1014. https://doi.org/10.1086 1285264

National Research Council of Canada. 2014. Sunrise/sunset calculator. Accessed 8 November 2013. http://www.nrc-cnrc .gc.ca/eng/services/hia/sunrise-sunset.html.

Rosenberg, K. V., and P. J. Blancher. 2005. Setting numerical population objectives for priority landbird species. Pages 57-67 in Bird Conservation Implementation and Integration in the Americas: Proceedings of the Third International Partners in Flight Conference. US Department of Agriculture, Forest Service General Technical Report PSW-GTR-191.

Scott, J. M., and F. L. Ramsay. 1981. Effects of abundant species on the ability of observers to make accurate counts of birds. Auk 98: 610-613.

Sexton, K., M. T. Murphy, L. J. Redmond, and A. C. Dolan. 2007. Dawn song of eastern kingbirds: intrapopulation variability and sociobiological correlates. Behaviour 144: 12731295. https://doi.org/10.1163/156853907781890922

Smith, S. M. 1991. The Black-capped Chickadee: Behavioral Ecology and Natural History. Comstock, New York, New York, USA.

Staicer, C., D. Spector, and A. Horn. 1996. The dawn chorus and other diel patterns in acoustic signaling. Pages 426453 in Ecology and Evolution of Acoustic Communication in Birds. Edited by D. E. Kroodsma and E. H. Miller. Cornell University Press, Ithaca, New York, USA.

Stoehr, A. M., and G. E. Hill. 2000. Testosterone and the allocation of reproductive effort in male house finches (Carpodacus mexicanus). Behavioral Ecology and Sociobiology 48: 407-411. https://doi.org/10.1007/s002650000247

Suter, S. M., D. Ermacora, N. Rieille, and D. R. Meyer. 2009. A distinct reed bunting dawn song and its relation to extrapair paternity. Animal Behaviour 77: 473-480. https: //doi.org/10.1016/j.anbehav.2008.11.002

Thogmartin, W. E. 2010. Sensitivity analysis of North American bird population estimates. Ecological Modelling 221: 173-177. https://doi.org/10.1016/j.anbehav.2008.11.002

Thompson, W. L. 2002. Towards reliable bird surveys: accounting for individuals present but not detected. Auk 119: 18-25. https://doi.org/10.2307/4090008

Urfi, A. J., M. Sen, A. Kalam, and T. Meganathan. 2005. Counting birds in India: methodologies and trends. Current Science 89: 1997-2003.

van Dongen, W. F. 2008. Mate guarding and territorial aggression vary with breeding synchrony in golden whistlers (Pachycephala pectoralis). Naturwissenschaften 95: 537545. https://doi.org/10.1007/s00114-008-0356-1

Ziolkowski Jr., D. J., K. L. Pardieck, and J. R. Sauer. 2010. On the road again for a bird survey that counts. Birding 42: 32-41.

Received 19 April 2016

Accepted 8 November 2016 